\title{
Ultraviolet Spectroscopy of a Coronal Mass Ejection
}

\author{
A. Ciaravella ${ }^{1}$, J.C. Raymond, S. Fineschi, L. Gardner, J. Michels, R. \\ O'Neal and J. Kohl
}

Harvard-Smithsonian Center for Astrophysics, 60 Garden St., Cambridge, $M A 02138$

M. Romoli and G. Noci

Dipartimento di Astronomia e Scienza dello Spazio, Università di Firenze, Largo E. Fermi 5, 50125 Firenze, Italy

C. Benna and S. Giordano

Università di Torino, I-10125 Torino, Italy

E. Antonucci

Osservatorio Astronomico di Torino, 10125 Pino Torinese, Italy

\begin{abstract}
A coronal mass ejection (CME) event was observed on December 23, 1996 with the Ultraviolet Coronagraph Spectrometer in both ultraviolet and visible light channels at $0.5 \mathrm{R}_{\odot}$ over the solar limb. The CME was followed during its evolution in the bright lines of Ly $\alpha$ (1216 $)$, $\operatorname{Ly} \beta(1026 \AA), \operatorname{Ly} \gamma(972 \AA), C$ III $(977 \AA)$ and the OVI doublet (1032, $1037 \AA)$ and in several weaker lines. The Ly $\alpha$ peak intensity shows an excursion of two orders of magnitude during the CME evolution, and blue shifts up to $0.8 \AA(\sim 200 \mathrm{~km} / \mathrm{sec})$. The data provide the emission measure in the Log $\mathrm{T}$ range $4.0-5.5$ with a 0.3 sampling. Line intensities and profiles have been measured, providing important diagnostics for a detailed study of the CME's physical and dynamical parameters.
\end{abstract}

\section{Introduction}

Statistical analysis of Coronal Mass Ejection (CME) observations has shown a correlation of these events with prominence eruptions. This has been confirmed by the Solar and Heliospheric Observatory (SOHO-Domingo et al. 1996) which, for the first time, observes CMEs from the onset region into the extended corona in several spectral bands. In particular, the Ultraviolet Coronagraph Spectrometer (UVCS) (Kohl et al. 1997, Noci et al. 1997, Antonucci et al. 1997a) has observed, at ultraviolet wavelengths, several CME events. In this paper we describe one of the first UVCS observations of a CME with preliminary results of its analysis (Antonucci et al. 1997b, Ciaravella et al. 1997).

\footnotetext{
${ }^{1}$ Also at: European Space Agency, ESA/Estec, Noordwijk, The Netherlands
} 


\section{The Instrument}

The Ultraviolet Coronagraph Spectrometer consists of two UV channels and a broadband visible light polarimeter designed to observe the solar corona from 1.4 to $11 \mathrm{R}_{\odot}$ (Kohl et al. 1995). The solar corona can be scanned through an entrance slit 40 ' long with a width variable in the range from a few to 84 ". The field of view of Visible light channel is a portion of $10 \times 10$ " located at center of the entrance slit.

The visible light polarimeter detects the linearly polarised intensity of the solar corona in the wavelength band $4500-6000 \AA$.

The UV channels are: the Ly $\alpha$ channel (LYA) optimised for measurement of Ly $\alpha 1215.67 \AA$ covers the range $1160-1350 \AA$; and the OVI channel (OVI) optimised for OVI doublet 1031.91 and $1037.61 \AA$ covers the range $945-1123 \AA$ (473-561 in the second order). In this channel lines in the range 1160-1270 (580-635 in the second order) can be also imaged. The dispersions are 0.1437 and $0.0993 \AA /$ pix in LYA and OVI detector, respectively.

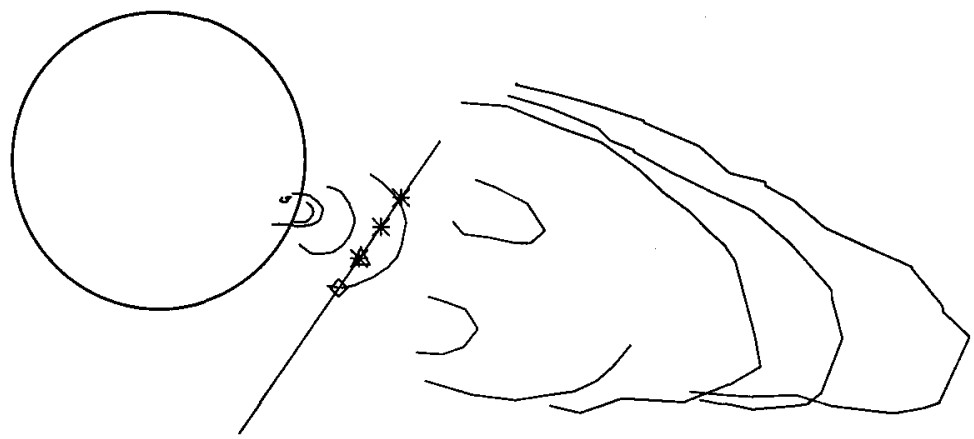

Figure 1. CME evolution through the field of view of EIT, C1 and C2/LASCO coronagraphs and the UVCS entrance slit position. The square marks the slit center and the asterisks mark the position of bright knots observed in the Ly $\alpha$ line.

\section{Observations}

The CME happened on December 23, 1996 in the Southwest. The event was observed with UVCS, the Extreme Ultraviolet Imaging Telescope (EIT) (Delaboudiniere et al. 1995) and the Large Angle Spectrometric Coronagraph (LASCO) (Brueckner et al. 1995). In Figure 1 is drawn the CME evolution as observed with EIT and LASCO and the UVCS entrance slit. The EIT $195 \AA$ images display the evolution of the prominence as the eruption causes a shock wave surrounding a dark void and the ejected plasma (Dere et al. 1997).

The UVCS observed at $235^{\circ} \mathrm{PA}$ at a fixed heliocentric height of $1.5 \mathrm{R}_{\odot}$ from 20:48:34 to 22:59:05 UT with 5 min exposures. In the LYA channel three spectral windows were imaged $1240.90-1243.49 \AA, 1210.51-1221.43 \AA$ and $1195.35-$ 
$1197.94 \AA$ binned to $0.29,0.14$ and $0.86 \AA$, respectively. The grating position was 120000 and the entrance slit width $50 \mu \mathrm{m}$ (14"). In the OVI channel we used four grating positions corresponding to $940-1040 \AA, 949-1049 \AA, 1017-1117 \AA, 1020-$ $1120 \AA$, wavelength ranges. The spectral binning was $0.2 \AA$. The spatial binning was 3 pixels (21") in the LYA channel and 10 pixels (70") in OVI.

In Figure 2 the Ly $\alpha 1215.67 \AA$ line is shown for two stages during the observation. The vertical axis gives the coordinate along the entrance slit and the zero marks the heliocentric distance of $1.5 \mathrm{R}_{\odot}$.
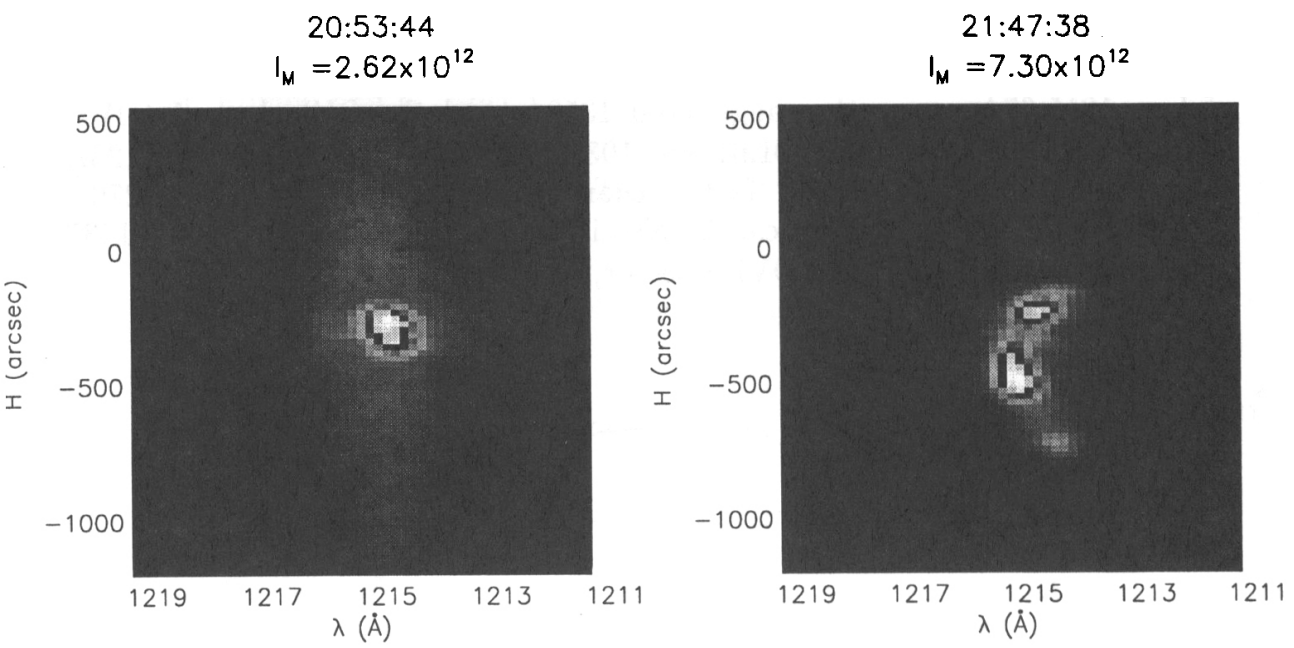

Figure 2. Two panels show the Ly $\alpha$ line emission at two times during the observation. The initial UT time of each exposure and the line peak intensity are shown. The vertical axis is the spatial coordinate along the entrance slit. Each image is scaled to its brightest pixel.

The initial UT time of each exposure and the line peak intensity are shown. The event occured in a streamer region. The bright knots detected in the Ly $\alpha$ line correspond to the prominence material ejected into the corona as a consequence of the eruption detected with EIT (Dere et al. 1997). The first panel shows the first evidence of the CME, the bright spot slightly blue-shifted with respect to the underlying streamer emission. Fifteen minutes later, at 21:09:57 UT, the CME was at the peak if its emission, about 500 times the original streamer emission. After this time the CME started to fade, and in about 1 $\mathrm{hr}$ and $30 \mathrm{~min}$ the initial streamer conditions were almost recovered. During the observation several bright knots appeared in the UVCS field of view (second panel in Figure 2).

The spectrum observed in the OVI detector at the same time (21:47:38 UT) is shown in Figure 3 . The O VI doublet, $\operatorname{Ly} \beta$, Si XII ( $499 \AA$ in second order), C III $(977 \AA)$ and Ly $\gamma$ can be seen. In spite of the coarse spatial binning used in this detector (70") the structure of CME material can still be seen. The three bright knots in C III show the same Doppler shifted structure as the Ly $\alpha$ line. The upper and lower knots are blue shifted as compared with the central one. 


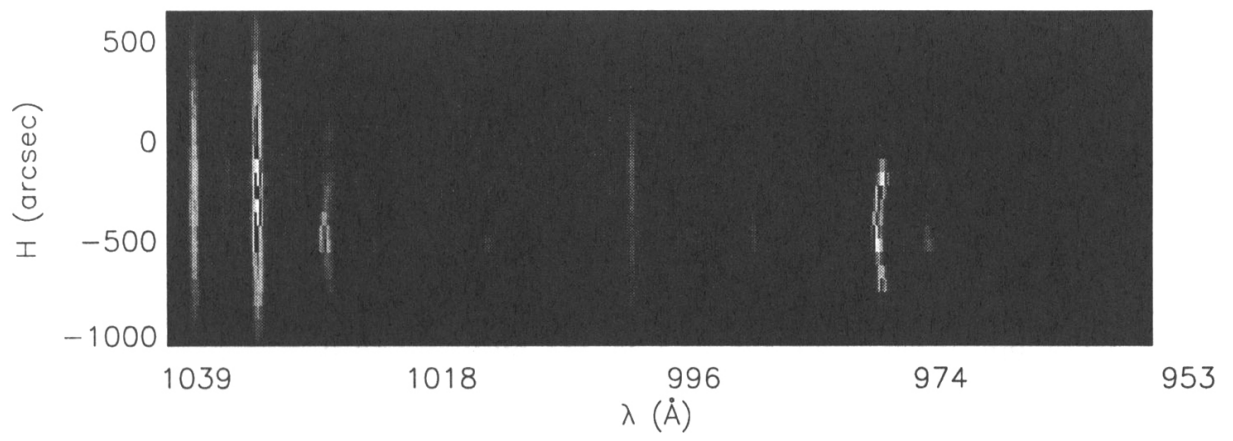

Figure 3. Spectrum observed in the OVI detector at the same time as panel 2 in Figure 2.

\section{Results}

\subsection{Emission Lines}

Several emission lines were observed during the CME evolution and most of them are low temperature lines which are normally not observed in the solar corona. A list of the brightest is shown in Table 1.

Table 1. Brightest Observed Lines

\begin{aligned} & \hline$\lambda_{I D}$ Identification \\ & \hline 629.73 O V $2 s^{2}{ }^{1} S_{0}-2 s 2 p{ }^{1} P_{1} \\ & 949.74$ Ly $\delta \\ & 972.54$ Ly $\gamma \\ & 977.02$ C III $2 s^{2}{ }^{1} S_{0}-2 s 2 p{ }^{1} P_{1} \\ & 989.79$ N III $2 s^{2}{ }^{2} P_{1 / 2}-2 s 2 p^{2}{ }^{2} D_{3 / 2} \\ & 991.58$ N III $2 s^{2}{ }^{2} P_{3 / 2}-2 s 2 p^{2}{ }^{2} D_{5 / 2} \\ & 1025.72$ Ly $\beta \\ & 1031.91$ O VI $2 s^{2} S_{1 / 2}-2 p^{2} P_{3 / 2} \\ & 1037.61$ O VI $2 s^{2} S_{1 / 2}-2 p^{2} P_{1 / 2} \\ & 1084.56$ N II $2 s^{2} 2 p^{2}{ }^{2} P_{1}-2 s 2 p^{3} D_{5 / 2} \\ & 1215.67$ Ly $\alpha \\ & 1242.80$ N V $2 s^{2} S_{1 / 2}-2 p{ }^{2} P_{1 / 2} \\ &$\hline\end{aligned}

\subsection{Temperature Estimate}

The spectral width of the bright knots observed in Ly $\alpha$ (Figure 2) is narrower than in the underlying streamer. We find in the bright knots of Ly $\alpha$ a Gaussian width narrower than in the streamer region, implying a temperature of ejected plasma at least six time lower than in the underlying streamer. Similar behavior has been found for the $\operatorname{Ly} \beta$, Ly $\gamma$ and $\operatorname{Ly} \delta$ lines.

Since C III $977.02 \AA$ and Ly $\beta$ show the same line widths, an upper limit to the plasma temperature of $7.4 \times 10^{4} \mathrm{~K}$ is obtained. On the other hand, using the 
Lyman line ratios and the C II to Ly $\alpha$ ratio two independent estimates of the plasma temperature can be obtained. The two approaches give different values. This result suggests that the hypothesis of plasma optically thin to the Lyman lines is not appropriate (Ciaravella et al. 1997).

\subsection{Velocity Structure}

The velocity field in the CME region appears very structured. In Figure 2, panel 2 , the lower and upper knots are blue shifted by 0.40 and $0.29 \AA$ with respect to the central one. During the observation we detected Ly $\alpha$ blue-shifts up to $0.8 \AA$ and red-shifts up to $0.2 \AA$, corresponding to line of sight velocities of 200 $\mathrm{km} / \mathrm{sec}$ and $50 \mathrm{~km} / \mathrm{sec}$, respectively. The CME extension along the slit increase from 200" at 20:53:44 UT to about 1000" at 21:47:38 UT implying expansion of about $200 \mathrm{~km} / \mathrm{sec}$. The bright knots have also a component of velocity along the UVCS slit. The average drift velocities are 70 and $50 \mathrm{~km} / \mathrm{sec}$ for the upper and middle knot in Figure 2.

\subsection{Emission Measure}

Using the brightest spectral lines we estimate the emission measure:

$$
\int n_{e} n_{H} d h=\frac{I_{\lambda}}{\left.<P_{\lambda}\right\rangle} \quad c m^{-5}
$$

where

$$
<P_{\lambda}>=\frac{P_{\max }}{2} \quad \text { phot } \mathrm{cm}^{3} \mathrm{sec}^{-1}
$$

The emission measure as a function of temperature for four times during the CME evolution is shown in Figure 4.

Except for the initial rapid decrease between 4.1 and 4.5 in $\log T$, the distribution is relatively flat as a function of temperature. The emission measures at $1.5 \mathrm{R}_{\odot}$ are three or four orders of magnitude smaller than EUV spectra of prominences in the literature (Schmahl and Orrall 1979). The C III $972 \AA$ and N III $991 \AA$ lines are formed at nearly the same temperature in ionization equilibrium, yet they imply emission measures differing by a factor of 3-4. An abundance anomaly related to the FIP effect (e.g., Meyer 1985) might cause such a discrepancy, as the material we observe was presumably well above the solar surface before the prominence erupted. Alternatively, the assumption of ionization equilibrium may be at fault.

\section{Conclusions}

A CME associated with a prominence eruption has been observed at UV wavelengths. The eruption occurred in a streamer region. Several low temperature lines were detected which are normally absent in a streamer region. The Lyman line widths suggest a temperature of ejected plasma at least six times lower than in the underlying streamer. The Ly $\alpha$ Doppler shifts give a diagnostic of the line of sight velocity structure. The Lyman line ratios suggest that the optically thin approximation is not valid to model the Lyman line intensities. Discrepancies in the emission measures of C III and N III lines were found. 


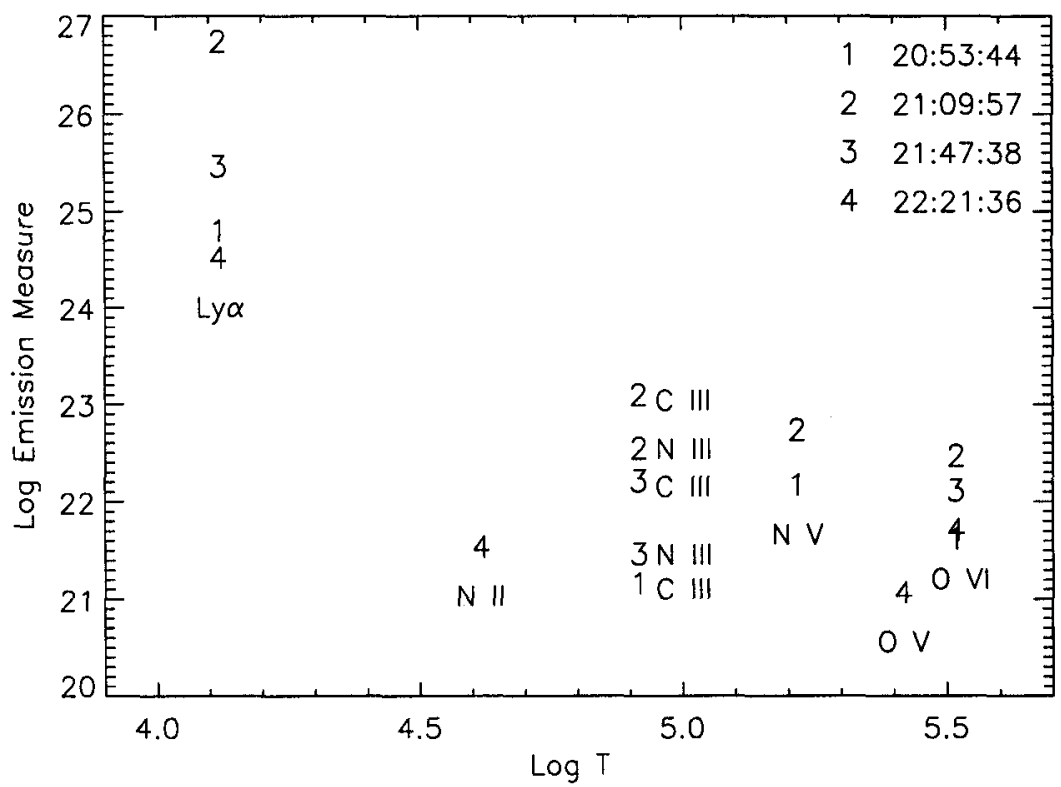

Figure 4. Emission measure as a function of temperature. The four symbols represent four stages of the CME evolution. At 21:09:57 (symbol 2) the CME was at the peak of its emission

\section{References}

Antonucci, E. et al. 1997a, Adv. Space Res., in press

Antonucci, E. et al. 1997b, ApJL, 490, 183.

Brueckner, G.E. et al. 1995, Sol. Phys, 162, 357

Ciaravella, A. et al. 1997, ApJL, 491, 59.

Delaboudiniere, J.P. et al. 1995, Sol. Phys, 162, 291

Dere, K.P. et al. 1997, Sol. Phys., 175, 601

Domingo, V., Fleck, B. and Poland, A. 1996, Sol. Phys., 162, 1

Kohl, J.L. et al. 1995, Sol. Phys, 162, 313

Kohl, J.L. et al. 1997, Sol. Phys., 175, 613

Meyer, J.P. 1985, ApJS, 57, 173

Noci, G. et al. 1997, Adv. Space Res., 20 (12), 2219

Schmahl, E.J. and Orrall, F.Q. 1979, ApJL, 231, L41 